\title{
Force-Velocity Assessment of Caress-Like Stimuli Through the Electrodermal Activity Processing: Advantages of a Convex Optimization Approach
}

\author{
Alberto Greco, Member, IEEE, Gaetano Valenza, Member, IEEE, Mimma Nardelli, Student Member, IEEE, \\ Matteo Bianchi, Member, IEEE, Luca Citi, Member, IEEE, and Enzo Pasquale Scilingo, Member, IEEE
}

\begin{abstract}
We propose the use of the convex optimization-based EDA (cvxEDA) framework to automatically characterize the force and velocity of caressing stimuli through the analysis of the electrodermal activity (EDA). CvxEDA, in fact, solves a convex optimization problem that always guarantees the globally optimal solution. We show that this approach is especially suitable for the implementation in wearable monitoring systems, being more computationally efficient than a widely used EDA processing algorithm. In addition, it ensures low-memory consumption, due to a sparse representation of the EDA phasic components. EDA recordings were gathered from 32 healthy subjects ( 16 females) who participated in an experiment where a fabric-based wearable haptic system conveyed them caress-like stimuli by means of two motors. Six types of stimuli (combining three levels of velocity and two of force) were randomly administered over time. Performance was evaluated in terms of execution time of the algorithm, memory usage, and statistical significance in discerning the affective stimuli along force and velocity dimensions. Experimental results revealed good performance of cvxEDA model for all of the considered metrics.
\end{abstract}

Index Terms-Affective haptics, caressing stimuli, convex optimization, CT fibers, electrodermal activity, electrodermal response, sparse representation, wearable haptics.

\section{INTRODUCTION}

W EARABLE haptics refers to a novel concept of haptic devices which can be easily worn by humans. A wearable haptic system applying stimuli to the human body can be used to allow augmented communication, interaction, and coop-

Manuscript received October 1, 2015; revised January 19, 2016, March 24, 2016, and May 4, 2016; accepted May 18, 2016. This work was supported in part by the European Union Seventh Framework Program FP7/2007-2013 under Grant 601165 of the project WEARHAP and in part by the European Research Council under the ERC Advanced Grant 291166 SoftHands (A Theory of Soft Synergies for a New Generation of Artificial Hands). This paper was recommended by Associate Editor J. Civera

A. Greco, G. Valenza, M. Nardelli, and E. P. Scilingo are with the Department of Information Engineering and Research Center "E. Piaggio," School of Engineering, University of Pisa, 56122 Pisa, Italy (e-mail: alberto.greco@ centropiaggio.unipi.it; g.valenza@iet.unipi.it; mimma.nardelli@for.unipi.it; e.scilingo@ing.unipi.it).

M. Bianchi is with the Department of Information Engineering and Research Center "E. Piaggio," School of Engineering, University of Pisa, 56122 Pisa, Italy, and also with the Advanced Robotics Department, Istituto Italiano di Tecnologia, 16163 Genova, Italy (e-mail: matteo.bianchi@centropiaggio.unipi.it).

L. Citi is with the School of Computer Science and Electronic Engineering, University of Essex, Colchester, CO4 3SQ, U.K. (e-mail: 1citi@essex.ac.uk).

Color versions of one or more of the figures in this paper are available online at http://ieeexplore.ieee.org.

Digital Object Identifier 10.1109/THMS.2016.2586478 eration with other robots, other humans, or other digital contents and virtual reality [1].

An emerging field of application of wearable haptics systems is the so-called "affective haptics" [2], [3]. Affective haptics refers to the ability of haptic systems to communicate emotions, possibly affecting social behavior and interactions [4]. This is possible because of the action of a specialized kind of tactile sensors in the skin, i.e., the unmyelinated CT tactile fibers [5], [6], whose activity is linked to the controlateral primary and bilateral secondary somatosensory area, as well as contralateral middle and posterior insula cortex [7]. Previous studies demonstrated how these fibers are sensitive to changes in the physical characteristics of the haptic stimulus. Specifically, changes in contact force and velocity of human caresses can vary the valence perception (pleasantness/unpleasantness) of the stimulus [8], [9]. Caressing stimuli having velocity of $3 \mathrm{~cm} / \mathrm{s}$ and applied forces as low as $2 \mathrm{~N}$ have been shown to convey a maximal pleasantness perception [8], thus optimally activating the CT fibers [10]. In addition, other physical parameters, such as texture and roughness, were found to have a role in the affective perception of the tactile stimulus [11].

In this study, we focus on the effects of affective haptic stimuli on autonomic nervous system (ANS) dynamics. Brain areas activated by CT fibers, in fact, are known to be also responsible for crucial homeostatic functions involving ANS activity from the whole body. More specifically, prior art reports on a strong correlation between ANS dynamics, as estimated through electrodermal activity (EDA) processing, and stressful and affective elicitations [4], [12]-[14].

It is well known from the literature that EDA signaling is comprised of a tonic component, having low-frequency information, and a phasic component, having higher frequency information [15], [16]. Anatomically, EDA is directly linked to the eccrine sweat glands activity through the sudomotor nerve activity (SMNA) [15], and can be easily monitored through voltage/current measures between two fingers [15]. Importantly, in a previous endeavor, we developed a fabric-based sensorized glove able to continuously record the EDA [13].

EDA processing was employed in a number of applications involving the assessment of haptic perception, including interaction with robots in nursing context [17], detection of nociceptive pain in adults [18] and preterm newborn infants [19], and in assessing the so-called "rubber hand illusion" [20] and vibrotactile sensory substitution [21]. Furthermore, EDA was used to assess the emotions in interacting partners [4], and to investigate the 
effect of tactile stimulation in persistent vegetative state [22], and in social anxiety [23].

However, a methodological framework to assess the physical characteristics of caressing stimuli, being also suitable for implementation in embedded computing systems, is still missing. To this extent, we here propose the application of a computationally efficient EDA processing algorithm to automatically assess the velocity and force of caressing stimuli elicited through a wearable haptic device. Here, we demonstrate that this algorithm, which is called convex optimization-based EDA (cvxEDA) [24], is suitable for embedded computing of EDA processing. CvxEDA, in fact, is based on a sparse representation of the phasic components of EDA thus requiring low-memory consumption, and it has been recently validated and proven to be effective also in affective computing applications [24]. Although sparse representation of EDA phasic components was also proposed in other studies [25] where the use of overcomplete dictionaries leads to a nonconvex problem, cvxEDA employes a convex optimization approach, thus enabling to always find the globally optimal solution [24]. In our recent study, performance of the proposed approach was compared with a deconvolution method for EDA tonic and phasic components separation, i.e., the continuous deconvolution analysis (CDA) [26]. This specific comparison was justified by the fact that CDA is a widely used model-based approach for the decomposition of the EDA in its components, and it has been a source of inspiration for cvxEDA development [24].

Data used in this study were gathered from 32 healthy subjects, enrolled in the frame of the European project "WEARHAP" (WEARable HAPtics for humans and robots). Each subject underwent affective haptic elicitation through a previously developed wearable haptic device able to mimic the human caress [3]. Specifically, a layer of fabric was stretched by two motors, whose position and velocity determined the force and velocity of the simulated caress. In this way, the artificial caresses could be controlled and standardized in terms of force and velocity [3]. Six kind of stimuli, by the combination of three level of velocity and two levels of force, were randomly administered along time.

The rest of the paper is organized as follows: Section II reports on the experimental setup, giving also details on the wearable device used for the haptic stimulation, and signal processing methods; Section III summarizes results, and Section IV discusses the experimental findings.

\section{MATERIALS AND METHODS}

Changes in EDA are directly linked to the eccrine sweat gland activity, which is modulated by the ANS through the SMNA. Such changes can be related to both the psycho-physiological state of a person, and the interaction with exogenous events. The EDA is practically measured by means of the skin conductance (SC) and therefore hereinafter we will refer to SC in place of EDA. The SC signal is defined in the frequency band of $0-2 \mathrm{~Hz}$ [27] and can be split into a slowly varying component, the socalled tonic component, and a superposed phasic component [15], [28]-[30].
Tonic component corresponds to the baseline level of the SC signal (i.e., SC level), and reflects the person's general psychophysiological state, and his autonomic regulation [15].The phasic component, instead, is comprised of consecutive, often overlapped, responses, called SC responses (SCRs), to exogenous stimuli such as lights, sounds, smells. SCRs are defined as variations in the SC signal arising within a predefined response window (1 $-5 \mathrm{~s}$ after stimulus onset) [26]. Sometimes, even if no stimuli are presented, there are some innate responses that are called nonspecific SC responses (NsSCR). They have the same characteristics as stimulus-related SCRs, but are considered tonic measures because they occur in the absence of external stimuli and in the absence of artifacts such as movements and sights [31].

In this section, we briefly report on details on the cvxEDA and the CDA models, which are described in [24] and [32]. Note that these two methods are able to discern overlapping consecutive SCRs, likely to be present in case of an interstimulus interval shorter than the SCR recovery time.

\section{A. EDA Processing Using Convex Optimization-Based Electrodermal Activity Algorithm}

CvxEDA proposed a representation of the SCRs as the output of a linear time-invariant system to a sparse nonnegative driver signal. The model assumes that the observed SC $(y)$ is the sum of the phasic activity $(r)$, a slow tonic component $(t)$, and an additive independent and identically distributed zero-average Gaussian noise term $\epsilon$

$$
y=r+t+\epsilon .
$$

Physiologically plausible characteristics (temporal scale and smoothness) of the tonic input signal can be achieved by means of a cubic spline with equally spaced knots every $10 \mathrm{~s}$, an offset and a linear trend term

$$
t=B \ell+C d
$$

where $B$ is a tall matrix whose columns are cubic B-spline basis functions, $\ell$ is the vector of spline coefficients, $C$ is a $N \times 2$ matrix (where $N$ is the length of the SC time series) with $C_{i, 1}=1$ and $C_{i, 2}=i / N, d$ is a $2 \times 1$ vector with the offset and slope coefficients for the linear trend.

The phasic component is the result of a convolution between the SMNA $p$, and an impulse response $h(t)$ shaped like a biexponential Bateman function [32]-[34]

$$
h(t)=\left(e^{-\frac{t}{\tau_{1}}}-e^{-\frac{t}{\tau_{2}}}\right) u(t)
$$

where $\tau_{1}$ and $\tau_{2}$ are, respectively, the slow and the fast time constants of the phasic curve shape, and $u(t)$ is the unitary step function.

Taking the Laplace transform of (3) and then its discrete-time approximation with sampling time $\delta$ (using a bilinear transformation), we obtain an autoregressive moving average (ARMA) model (see details in [24]) that can be represented in matrix form as

$$
H=M^{-1} A
$$


where $M$ and $A$ are tridiagonal matrices with the MA and AR coefficients along the diagonals. Using an auxiliary variable $q$ such that

$$
q=A^{-1} p, \quad r=M q
$$

we write the final observation model as

$$
y=M q+B \ell+C d+\epsilon .
$$

Given the EDA model (6), the goal is to identify the maximum a posteriori (MAP) neural driver SMNA $(p)$ and tonic component $(t)$ parametrized by $[q, \ell, d]$, for the measured SC signal (y). CvxEDA rewrites the MAP problem as a constrained minimization QP convex problem (see details in [24])

$$
\begin{aligned}
\text { minimize } & \frac{1}{2}\|M q+B \ell+C d-y\|_{2}^{2}+\alpha\|A q\|_{1}+\frac{\gamma}{2}\|\ell\|_{2}^{2} \\
\text { subj. to } & A q \geq 0 .
\end{aligned}
$$

After some matrix algebra, this optimization problem can be rewritten in the standard QP form and solved efficiently using one of the many sparse-QP solvers available. After finding the optimal $[q, \ell, d]$, the tonic component $t$ can be derived from (2), while the SMNA driving the phasic component can be easily found as $p=A q$ (see also the upper diagram in Fig. 2).

The objective function (7) to be minimized is a quadratic measure of misfit or prediction error between the observed data and the values predicted by the model. Moreover, the prior knowledge about the spiking sparse nature and nonnegativity of the SMNA $(p)$ and the smoothness of the tonic component are accounted for by the regularizing terms and the constraint.

The strength of the penalty is regulated by $\alpha$ and $\gamma$ terms. A sparser estimate is yielded by large values of $\alpha$. Concerning $\gamma$, higher values mean a stronger penalization of $\ell$, i.e., a smoother tonic curve. Of note, fixed values of $\tau_{1}=0.7 \mathrm{~s}$, $\tau_{2}=3.0 \mathrm{~s}, \alpha=0.4$, and $\gamma=0.01$, which were chosen during previous exploratory tests on separate data, were employed throughout this analysis.

CvxEDA algorithm is implemented in MATLAB language and the software is available online (http://www.mathworks. com/matlabcentral/fileexchange/53326-cvxeda).

\section{B. EDA Processing Using CDA}

CDA [15] also describes the relationship between SMNA and SC data using the Bateman function, which has already been described in the previous paragraph [see (3)]

$$
\operatorname{EDA}(t)=\operatorname{SMNA}(t) \otimes h(t)
$$

where SMNA $=\left(\right.$ DRIVER $_{\text {tonic }}+$ DRIVER $\left._{\text {phasic }}\right)$.

In (8), SMNA is the unknown quantity that is estimated by deconvolving the EDA signal with $h(t)$.

The CDA algorithm is comprised of three stages (see the bottom diagram in Fig. 2): the preprocessing stage, the decomposition of the SC in its tonic and phasic component, and the optimization stage.

The preprocessing consists of the visual detection and removal of the movement artifacts, and a low-pass zero-phase filtering with a $2 \mathrm{~Hz}$ cutoff. The decomposition in the two driver
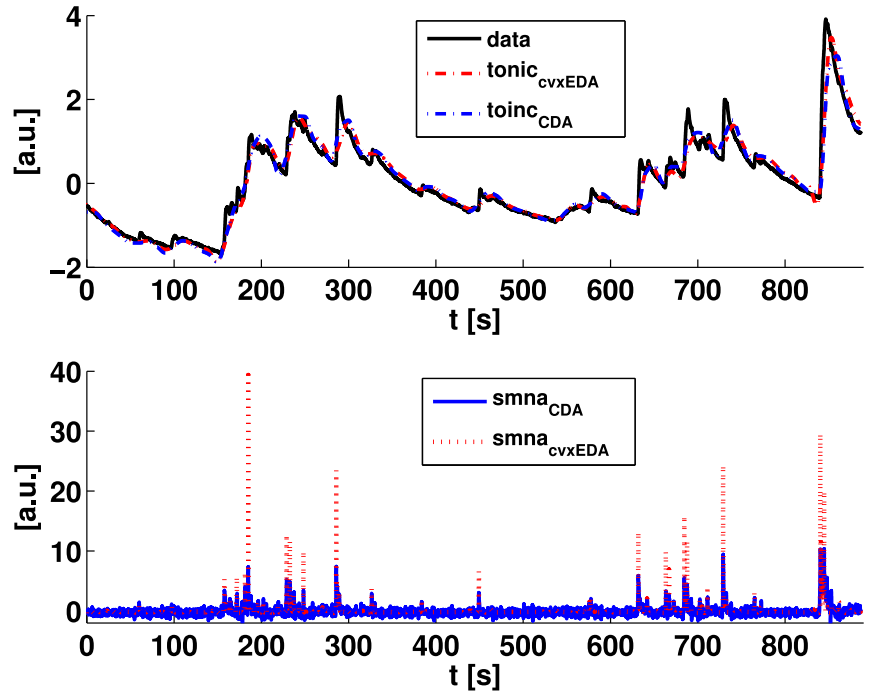

Fig. 1. Application of the cvxEDA (red) and CDA (blue) decomposition procedure to the SC signal recorded during the affective haptic stimulation.

functions is achieved by a deconvolution between the SC data and the impulse response function. The hypothesis underlying $\mathrm{SC}$ component behaviors is that tonic activity is observable in the absence of any phasic activity [15]. Therefore, the DRIVER tonic $_{\text {. }}$ component is obtained by the application of a smoothing Gauss window of $200 \mathrm{~ms}$ and a peak detection algorithm in order to find the peaks under a threshold of $0.2 \mu S$, used to build a 10-s spacing grid and then the grid points were interpolated with a cubic spline fitting method. The DRIVER phasic $_{\text {component, }}$ instead, is computed by subtracting the previously estimated DRIVER $_{\text {tonic }}$ from the SMNA.

Finally, the optimization stage aims at increasing the sparsity of the DRIVER phasic $_{\text {signal and obtain a signal with a }}$ zero baseline and peaks as distinguishable as possible. Starting from fixed values, the parameter $\tau_{1}$ and $\tau_{0}$ of $(h(t))$ are optimized minimizing a specific cost function given by the sum of

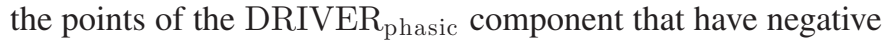
value and the points above a predefined threshold. More details can be again found in [32]. Note that the CDA algorithm is implemented in the Ledalab software package for MATLAB [35].

\section{Subject Recruitment, Experimental Protocol, and Acquisition Set-Up}

Thirty-two healthy subjects aged $27 \pm 2$ (16 males, age $26 \pm$ 2 , and 16 females, age $27 \pm 2$ ) gave their informed consent to take part in the study. This study was approved by the local Ethical Committee. Exemplary data from one subject are shown in Fig. 1. Caresses were administered through an affective touch display, described in detail in [3]. Specifically, subjects were asked to place the forearm on a support so as to put the upper side in contact with the fabric layer. The fabric is connected to two motors, whose rotations allow modifying velocity and force of the mimicked caress (see Fig. 3).

Throughout the experiment, participants wore earplugs in order to prevent any auditory cues, while their SC was recorded 

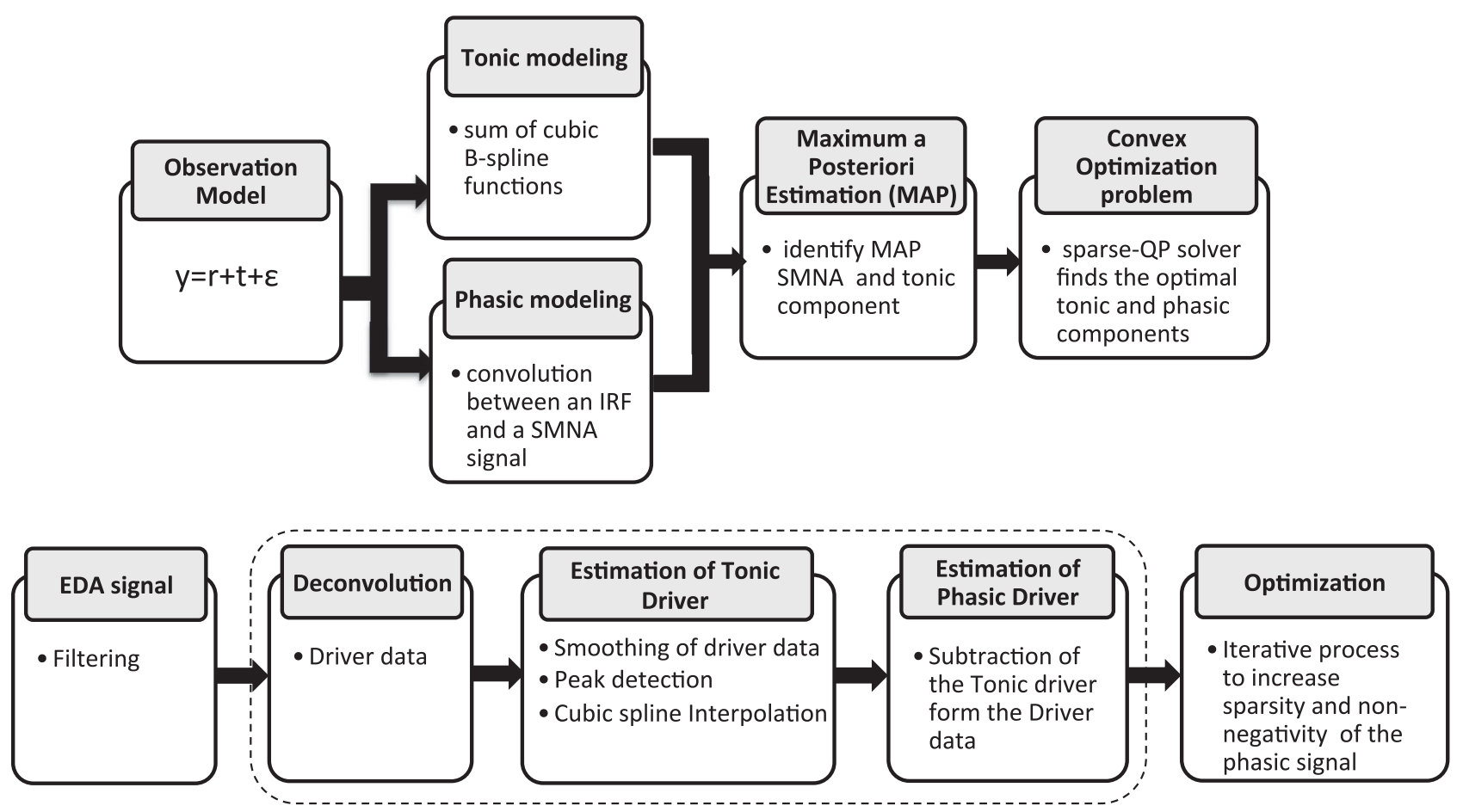

Fig. 2. Block diagrams of both cvxEDA (upper) and CDA (bottom) methods. They summarize the main steps of both processing chains.

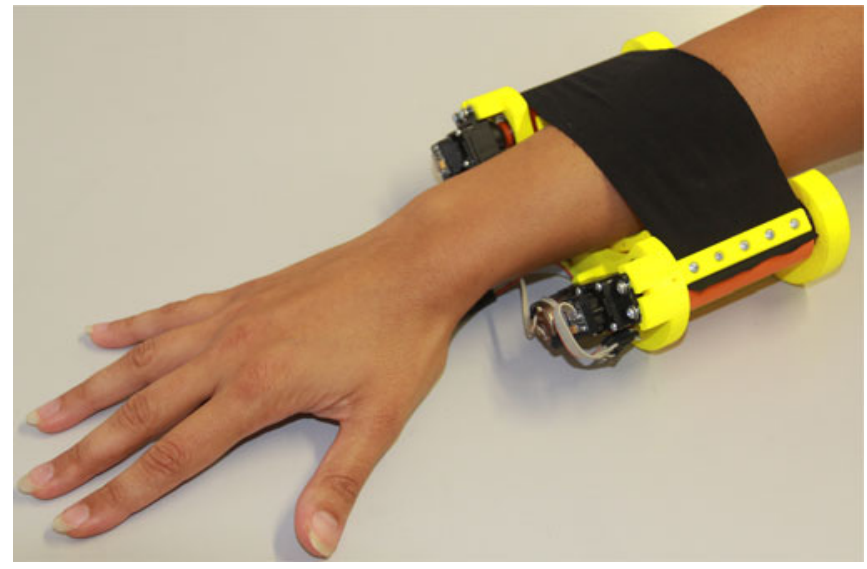

Fig. 3. Overview of the haptic system worn by a subject.

using a BIOPAC MP150 physiological acquisition system. Following the literature guidelines and in order to avoid interferences between the acquisition and the elicitation devices, haptic stimuli were applied on the dominant forearm, whereas the BIOPAC system measured the SC on the distal phalanxes of the index and middle finger of the nondominant hand. Prior to the experiment, the haptic device's load cell was calibrated with respect to the forearm weight. We used six different combinations of stimuli through two levels of force $(\mathrm{F} 1=2 \mathrm{~N}, \mathrm{~F} 2=6 \mathrm{~N})$ and three levels of velocity $(\mathrm{V} 1=9.4 \mathrm{~mm} / \mathrm{s}, \mathrm{V} 2=37 \mathrm{~mm} / \mathrm{s}$, and $\mathrm{V} 3=65 \mathrm{~mm} / \mathrm{s}$ ). In between two stimuli, the motors were stopped and the force was set to $0 \mathrm{~N}$. The values of velocity were chosen according to previous studies, which explored the hedonic aspects of caress-like stimulation on glabrous skin in a range between 0.1 and $30 \mathrm{~cm} / \mathrm{s}$. In such studies, the pleasantness

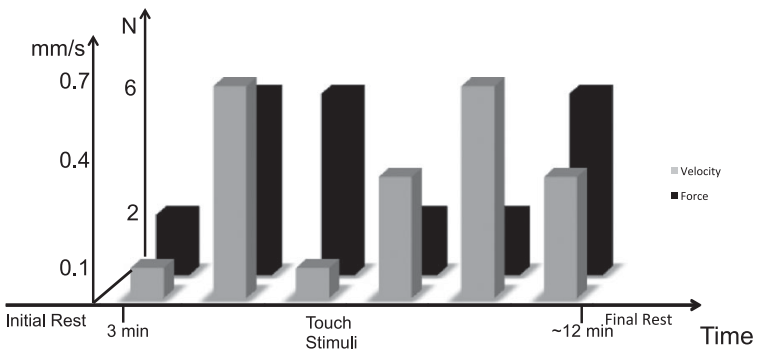

Fig. 4. Example of protocol scheme of one subject. Velocity and force combination is randomized.

ratings showed an inverted U-shaped pattern, with the highest values related to the range $1-3 \mathrm{~cm} / \mathrm{s}[8]-[10]$. Moreover, in our previous work, we analyzed the subjects' self-reports on the perceived emotive response to caress-like haptic stimuli which included different velocities and force levels [3].

The experimental protocol consisted of the following consecutive sessions:

1) resting state of $3 \mathrm{~min}$;

2) randomized caressing elicitation of $\sim 12 \mathrm{~min}$; and

3) resting state of $2 \mathrm{~min}$.

Of note, all the six combinations of velocities and forces were randomized among subjects (see an example of protocol scheme in Fig. 4), with a prestimulus and a poststimulus interval of 35 s each.

\section{Feature Extraction, Performance Metrics, and Statistical Analysis}

Once the tonic and phasic components are estimated from both cvxEDA and CDA approaches, several features can be 
TABLE I

List OF FEATURES EXTRACTED FROM EDA PHASIC AND TONIC COMPONENTS

\begin{tabular}{ll}
\hline \hline Phasic Feature & \multicolumn{1}{c}{ Description } \\
\hline Npeak & Number of significant SCR wrw \\
AUC & Area under curve of SMNA signal wrw $(\mu S s)$ \\
peak & Maximum amplitude of significant SCRs wrw ${ }^{1}(\mu S)$ \\
stdphasic & Standard deviation of SMNA signal wrw $(\mu S)$ \\
\hline Tonic Feature & Description \\
MeanTonic & $\begin{array}{l}\text { Mean value of the tonic component wrw }(\mu S) \\
\text { MeanTonic difference between }\end{array}$ \\
diffTonic & $\begin{array}{l}\text { post/prerest sessions }(\mu S) \\
\text { Difference of AUC of spontaneous SCRs }\end{array}$ \\
diffNSAUC & between post/prerest sessions $(\mu S s)$ \\
\hline \hline
\end{tabular}

$\mathrm{wrw}=$ within response window (i.e., $5 \mathrm{~s}$ after stimulus).

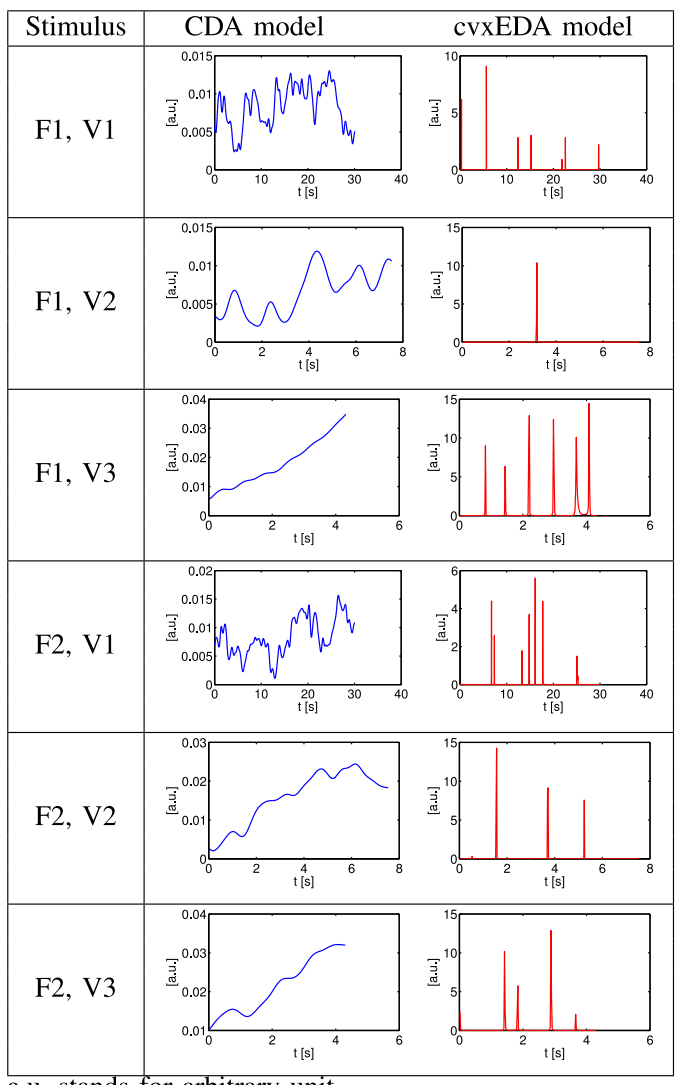

a.u. stands for arbitrary unit.

Fig. 5. Example of CDA (left) and cvxEDA (right) phasic responses to each combination of caressing force $(F=2 \mathrm{~N}, F=6 \mathrm{~N})$ and velocity (V1 $=9.4 \mathrm{~mm} / \mathrm{s}, \mathrm{V} 2=37 \mathrm{~mm} / \mathrm{s}$, and $\mathrm{V} 3=65 \mathrm{~mm} / \mathrm{s}$ ) level, within the stimulus time window.

extracted to investigate the sensitivity to changes in caressing, along the force and velocity dimensions. Proposed features in this study are summarized in Table I.

Through these features, the following analyses were performed.

1) Event-related phasic analysis, through which EDA was studied within a time response window of $5 \mathrm{~s}$ after the affective stimulus Fig. 5.

2) Nonspecific fluctuation and tonic analysis, comprised of a) the tonic value, averaged within the time-response

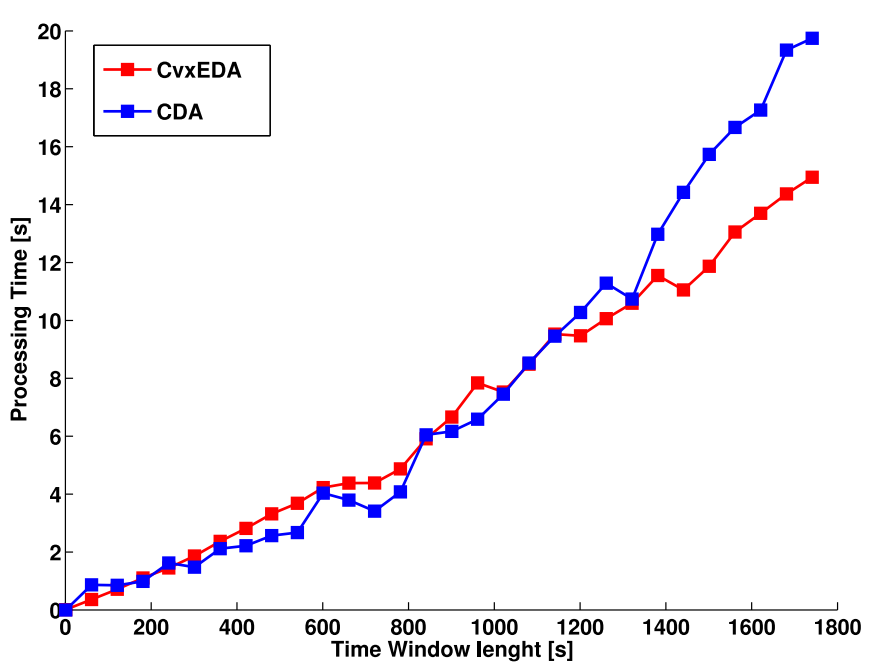

Fig. 6. Processing time of the CDA and cvxEDA algorithms at different length of signal input, with sampling time of $60 \mathrm{~s}$.
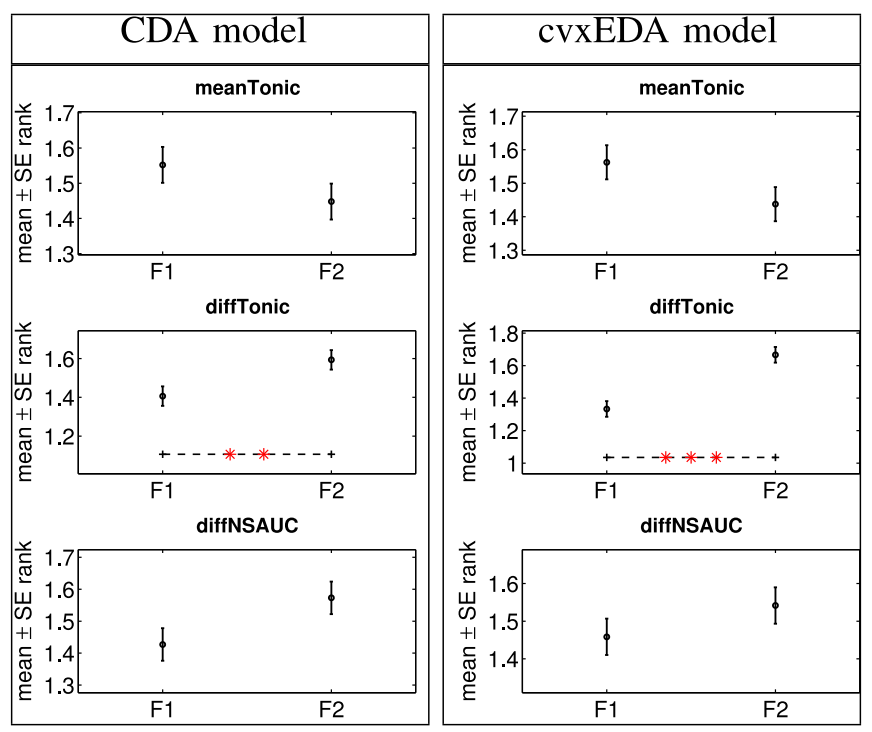

Fig. 7. Within-subject ranks of the tonic feature set obtained from CDA (left) and cvxEDA (right) models between the two force levels $(\mathrm{F} 1=2 \mathrm{~N}, \mathrm{~F} 2=6 \mathrm{~N})$ Values represent average rank \pm standard error (SE) across subjects. Asterisks indicate significant differences between velocities: $(*) p<0.05,(* *) p<0.01$, $(* * *) p<0.001$

window, and the nonspecific electrodermal fluctuations, and b) differential tonic value between the post- and prestimulus session.

The differences between the two levels of force (i.e., $\mathrm{F} 1=$ $2 \mathrm{~N}$ and $\mathrm{F} 2=6 \mathrm{~N}$ ) and among the three velocities (i.e., $\mathrm{V} 1=$ $9.4 \mathrm{~mm} / \mathrm{s}, \mathrm{V} 2=37 \mathrm{~mm} / \mathrm{s}$, and $\mathrm{V} 3=65 \mathrm{~mm} / \mathrm{s}$ ) were studied using Wilcoxon signed-rank tests and Friedman tests, respectively, due to the non-Gaussianity of samples (as confirmed by a preliminary analysis performed through Shapiro-Wilk tests). In case of rejection of the Friedman test null-hypothesis, a post hoc analysis, using Wilcoxon signed-rank with Bonferroni correction, was also carried out.

Finally, to test the suitability of the proposed algorithm in embedded computing systems, computational performance 
TABLE II

MEDIAN \pm MAD (MEDIAN ABSOLUTE DEVIATION) INTERVAL FOR CDA FEATURES

\begin{tabular}{lccc}
\hline \hline Feature & F1 & F2 & $p$-value \\
\hline Npeak & $0 \pm 0$ & $0 \pm 0$ & $>0.05$ \\
Peak & $0.0419 \pm 0.0255$ & $0.0387 \pm 0.0256$ & $>0.05$ \\
AUC & $0.236 \pm 0.176$ & $0.263 \pm 0.202$ & $>0.05$ \\
stdphasic & $0.0108 \pm 0.00891$ & $0.00996 \pm 0.00834$ & $>0.05$ \\
MeanTonic & $1.67 \pm 0.452$ & $1.55 \pm 0.413$ & $>0.05$ \\
diffTonic & $-0.0457 \pm 0.067$ & $-0.0113 \pm 0.0624$ & $<\mathbf{0 . 0 0 5}$ \\
diffNSAUC & $0.112 \pm 0.122$ & $0.151 \pm 0.156$ & $>0.05$ \\
\hline \hline
\end{tabular}

Values were averaged among the subjects. Last column shows $p$-values from Wilcoxon nonparametric tests, with null hypothesis of equal median values between two force levels.

Bold values mean statistically significant $\mathrm{p}$-value.

analysis was also performed. This is related to the execution time of the algorithm (processing time), and the memory usage of the CDA and cvxEDA models output vectors. Concerning the running time analysis, as a first step, we calculated the time to decompose $60 \mathrm{~s}$ of SC signal. Then, we incremented the time length of the SC segment with a sampling step of $60 \mathrm{~s}$, until we estimated the processing time needed to decompose the total length of the SC signal (1800 s).

Estimates of these metrics were obtained on the same personal computer, with processor $1.7 \mathrm{GHz}$ Intel Core i7, RAM memory 8 GB $1600 \mathrm{MHz}$ DDR3.

\section{EXPERIMENTAL RESULTS}

In this section, results from the statistical analysis performed on features from CDA and cvxEDA algorithms are reported. Such results are shown considering EDA nonspecific fluctuations and tonic components analysis, as well as event-related phasic components analysis.

\section{A. EDA Nonspecific Fluctuations and Tonic Components Analysis}

Only one feature, diffTonic, extracted from both the CDA and cvxEDA models showed a significant difference $(p<0.005$, Fig. 7) between the two levels of force while caressing. In particular, the higher the diffTonic values, the higher the caressing force. Note that diffTonic calculated through cvxEDA showed a higher discerning power, i.e., lower $p$-value, as compared with CDA modeling (see Tables II and III).

Concerning the three levels of caress velocity, among the features extracted using the CDA model, significant differences $(p<0.05)$ were found in diffTonic while discerning $V 1$ versus $V 2$, and $V 1$ versus $V 3$, and in diffNSAUC while discerning $V 1$ versus $V 3$ and $V 2$ versus $V 3$ (see Table IV and Figs. 9 and 10). On the other hand, using the cvxEDA model, a significant difference in discerning $V 1$ versus $V 2$ was found through meanTonic (see Table $V$ and Fig. 9). CDA showed a higher number of statistical differences among the velocities through the tonic features than cvxEDA, though a complete discrimination of all of the three levels is not achieved by both.
TABLE III

MEDIAN \pm MAD INTERVALS FOR CVXEDA FEATURES

\begin{tabular}{lccc}
\hline \hline Feature & F1 & F2 & $p$-value \\
\hline Npeak & $3.0 \pm 1.0$ & $3.0 \pm 1.0$ & $>0.05$ \\
Peak & $3.99 \pm 3.94$ & $3.78 \pm 3.58$ & $>0.05$ \\
AUC & $24.6 \pm 21.6$ & $32.0 \pm 27.5$ & $>0.05$ \\
stdphasic & $0.414 \pm 0.41$ & $0.412 \pm 0.378$ & $>0.05$ \\
MeanTonic & $-0.289 \pm 0.476$ & $-0.406 \pm 0.445$ & $>0.05$ \\
diffTonic & $-0.109 \pm 0.231$ & $0.0453 \pm 0.382$ & $<\mathbf{0 . 0 0 0 5}$ \\
diffNSAUC & $2.11 \pm 7.01$ & $4.9 \pm 10.1$ & $>0.05$ \\
\hline \hline
\end{tabular}

Values were averaged among the subjects. Last column shows $p$ values from Wilcoxon nonparametric tests, with null hypothesis of equal median values between two force levels.

Bold values mean statistically significant $\mathrm{p}$-value.

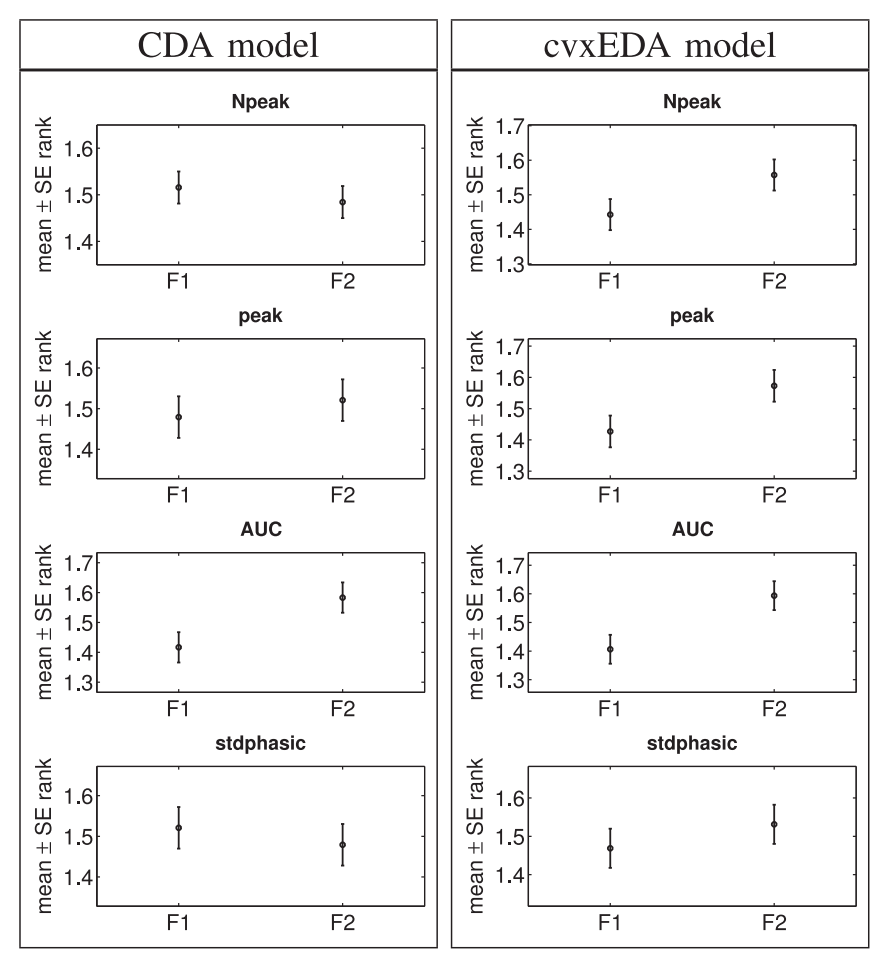

Fig. 8. Within-subject ranks of the phasic feature set obtained from CDA (left) and cvxEDA (right) models between the two force levels $(\mathrm{F} 1=2 \mathrm{~N}, \mathrm{~F} 2=6 \mathrm{~N})$. Values represent average rank \pm standard error (SE) across subjects. Asterisks indicate significant differences between velocities: $(*) p<0.05,(* *) p<0.01$, $(* * *) p<0.001$.

\section{B. EDA Event-Related Phasic Components Analysis}

This analysis revealed no significant differences between the two caressing forces, being consistent between the CDA and cvxEDA approaches (see Tables II and III, and Fig. 8).

Concerning differences between the three caressing velocities, CDA modeling showed significant differences $(p<0.002)$ on the number of significant peaks (Npeak), and the area under the phasic signal curve (AUC) while discerning $V 1$ versus $V 2$, and $V 1$ versus $V 3$. No significant differences were found otherwise, including $V 2$ versus $V 3$ (see Table IV and Fig. 10). Using the cvxEDA approach, instead, we found significant differences in all of the pairwise comparisons, with $p<0.005$ (see Table V and Fig. 10). Importantly, a monotonically 
TABLE IV

MEDIAN \pm MAD INTERVALS FOR CDA FEATURES

\begin{tabular}{|c|c|c|c|c|c|c|c|}
\hline \multirow[t]{2}{*}{ Feature } & \multirow[t]{2}{*}{ V1 } & \multirow[t]{2}{*}{ V2 } & \multirow[t]{2}{*}{ V3 } & \multicolumn{4}{|c|}{$p$-value } \\
\hline & & & & Friedman & $\mathrm{V} 1-\mathrm{V} 2$ & $\mathrm{~V} 1-\mathrm{V} 3$ & $\mathrm{~V} 2-\mathrm{V} 3$ \\
\hline Npeak & $0 \pm 0$ & $1.0 \pm 1.0$ & $1.0 \pm 1.0$ & $<1 \mathrm{e}-5$ & $<0.005$ & $<1 \mathrm{e}-5$ & $>0.05$ \\
\hline peak & $0.0519 \pm 0.0308$ & $0.0344 \pm 0.0216$ & $0.0397 \pm 0.0255$ & $>0.05$ & $>0.05$ & $>0.05$ & $>0.05$ \\
\hline AUC & $0.0964 \pm 0.0634$ & $0.331 \pm 0.241$ & $0.343 \pm 0.254$ & $<1 \mathrm{e}-14$ & $<1 \mathrm{e}-6$ & $<1 \mathrm{e}-8$ & $>0.05$ \\
\hline stdphasic & $0.0134 \pm 0.0108$ & $0.0103 \pm 0.0076$ & $0.00924 \pm 0.01773$ & $>0.05$ & $>0.05$ & $>0.05$ & $>0.05$ \\
\hline MeanTonic & $1.53 \pm 0.369$ & $1.69 \pm 0.467$ & $1.64 \pm 0.463$ & $>0.05$ & $>0.05$ & $>0.05$ & $>0.05$ \\
\hline diffTonic & $-0.0663 \pm 0.0815$ & $0.00352 \pm 0.0773$ & $-0.0175 \pm 0.0599$ & $<0.005$ & $<0.005$ & $<0.05$ & $>0.05$ \\
\hline diffNSAUC & $0.0633 \pm 0.0697$ & $0.127 \pm 0.148$ & $0.259 \pm 0.219$ & $<1 \mathrm{e}-5$ & $>0.05$ & $<0.005$ & $<0.001$ \\
\hline
\end{tabular}

Values were averaged among the subjects. Last four columns show $p$-values from the Friedman test and Wilcoxon nonparametric tests, with null hypothesis of equal median values between three velocity levels. Bold values mean statistically significant p-value.

TABLE V

MEDIAN \pm MAD INTERVALS FOR CVXEDA FEATURES

\begin{tabular}{|c|c|c|c|c|c|c|c|}
\hline \multirow[t]{2}{*}{ Feature } & \multirow[t]{2}{*}{ V1 } & \multirow[t]{2}{*}{$\mathrm{V} 2$} & \multirow[t]{2}{*}{ V3 } & \multicolumn{4}{|c|}{ p-value } \\
\hline & & & & Friedman & $\mathrm{V} 1-\mathrm{V} 2$ & $\mathrm{~V} 1-\mathrm{V} 3$ & $\mathrm{~V} 2-\mathrm{V} 3$ \\
\hline Npeak & $2.0 \pm 1.0$ & $3.0 \pm 1.5$ & $4.0 \pm 2.0$ & $<1 \mathrm{e}-5$ & $<0.005$ & $<1 \mathrm{e}-6$ & $<0.0005$ \\
\hline Peak & $0.256 \pm 0.256$ & $4.36 \pm 4.03$ & $7.61 \pm 6.16$ & $<1 \mathrm{e}-9$ & $<1 \mathrm{e}-5$ & $<1 \mathrm{e}-6$ & $>0.05$ \\
\hline AUC & $6.89 \pm 6.59$ & $32.4 \pm 24.8$ & $72.6 \pm 42.0$ & $<1 \mathrm{e}-12$ & $<1 \mathrm{e}-7$ & $<1 \mathrm{e}-8$ & $<1 \mathrm{e}-4$ \\
\hline stdphasic & $0.0356 \pm 0.0355$ & $0.469 \pm 0.406$ & $0.752 \pm 0.577$ & $<1 \mathrm{e}-11$ & $<1 \mathrm{e}-6$ & $<1 \mathrm{e}-7$ & $<0.05$ \\
\hline MeanTonic & $-0.527 \pm 0.471$ & $-0.261 \pm 0.429$ & $-0.292 \pm 0.568$ & $<0.05$ & $<0.05$ & $>0.05$ & $>0.05$ \\
\hline diffTonic & $-0.122 \pm 0.335$ & $-0.0251 \pm 0.303$ & $0.0417 \pm 0.221$ & $>0.05$ & $>0.05$ & $>0.05$ & $>0.05$ \\
\hline diffNSAUC & $2.1 \pm 6.61$ & $2.13 \pm 9.22$ & $5.43 \pm 9.37$ & $>0.05$ & $>0.05$ & $>0.05$ & $>0.05$ \\
\hline
\end{tabular}

Values were averaged among the subjects. Last four columns show $p$-values from the Friedman test and Wilcoxon nonparametric tests, with null hypothesis of equal median values between three velocity levels.

Bold values mean statistically significant p-value.

TABLE VI

MEDIAN \pm MAD INTERVALS FOR CDA AND CVXEDA PERFORMANCES

\begin{tabular}{lccc}
\hline \hline & & & \\
& CDA & cvxEDA & $p$-value \\
\hline Processing time [s] & $8.171 \pm 1.239$ & $7.038 \pm 1.657$ & $>0.05$ \\
Memory [Kb] & $359.004 \pm 2.868$ & $136.65 \pm 95.384$ & $<\mathbf{0 . 0 0 1}$ \\
\hline \hline
\end{tabular}

p-values are gathered from the Mann-Whitney nonparametric tests with null hypothesis of equal medians between models. Values were calculated for each recording, and averaged among the subjects.

Bold values mean statistically significant p-value.

increasing trend among the three velocities was found for each of the considered features (see Fig. 10). Fig. 5 shows exemplary EDA phasic responses from CDA and cvxEDA models, for each combination of caressing force and velocity. Note that, considering sparse outputs from cvxEDA, amplitude values are consistent with the caressing velocity level.

\section{Computational Performance Analysis}

Results from the computational performance analysis, showing groupwise statistics, are reported in Table VI.

Concerning the processing time, no significant difference was found between the two models. However, as we show in Fig. 6, CDA execution time is not linearly related to the length of the input signal. In particular, for input signals longer than
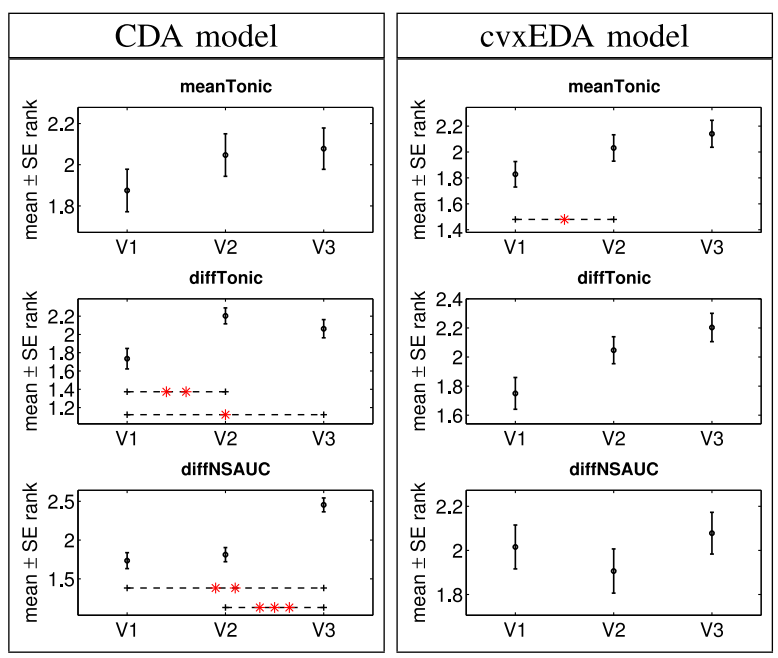

Fig. 9. Within-subject ranks of the tonic feature set obtained from CDA (left) and cvxEDA (right) models between the three velocity levels (V1 $=9.4 \mathrm{~mm} / \mathrm{s}$, $\mathrm{V} 2=37 \mathrm{~mm} / \mathrm{s}$ and $\mathrm{V} 3=65 \mathrm{~mm} / \mathrm{s}$ ). Values represent average rank \pm standard error (SE) across subjects. Asterisks indicate significant differences between velocities: $(*) p<0.05,(* *) p<0.01,(* * *) p<0.001$.

1300 s, CDA processing time tends to grow superlinearly. Concerning memory usage, a significant difference was found between the two models. As expected, given to the intrinsic sparse 


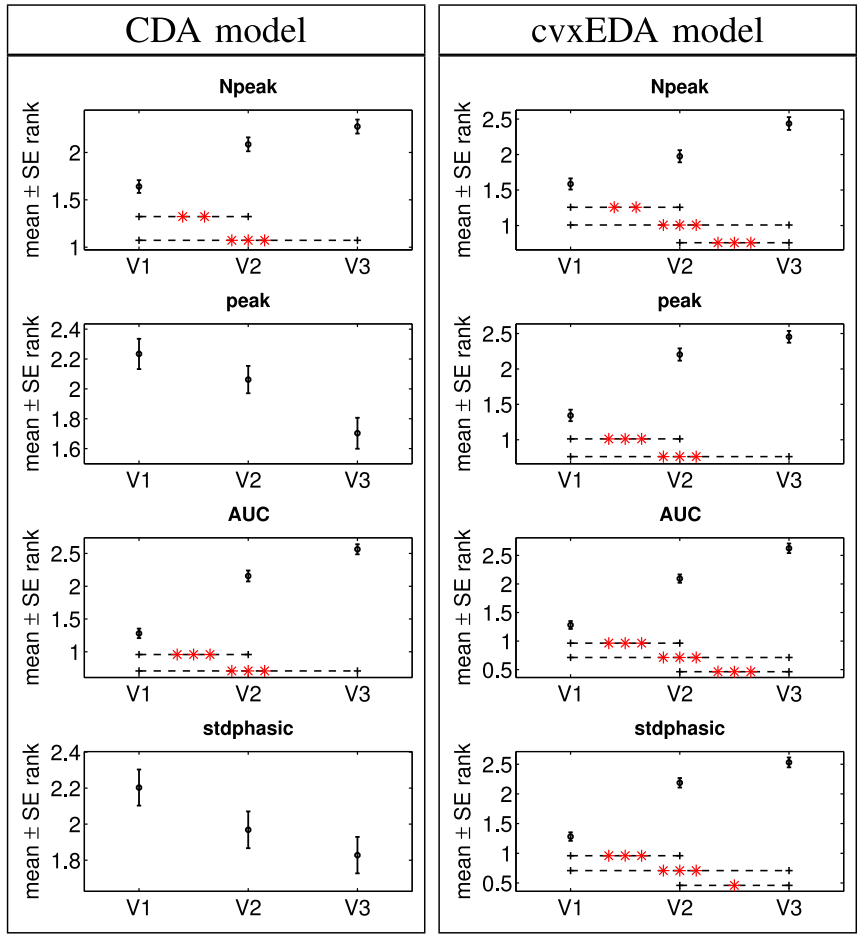

Fig. 10. Within-subject ranks of the phasic feature set obtained from CDA (left) and cvxEDA (right) models between the three velocity levels (V1 = $9.4 \mathrm{~mm} / \mathrm{s}, \mathrm{V} 2=37 \mathrm{~mm} / \mathrm{s}$, and $\mathrm{V} 3=65 \mathrm{~mm} / \mathrm{s}$ ). Values represent average rank \pm standard error (SE) across subjects. Asterisks indicate significant differences between velocities: $(*) p<0.05,(* *) p<0.01,(* * *) p<0.001$.

nature of cvxEDA phasic components, lower storage values were associated with the cvxEDA model.

\section{CONCLUSION AND DisCUSSION}

In this paper, we proposed the cvxEDA approach [24] to perform EDA processing through wearable monitoring systems, during affective haptic stimulation. In the literature, several methods for the analysis of the EDA have been presented, such as "continuous deconvolution analysis" [32], PsPM suite [36], [37], Lim et al. model [38] or Chaspari et al. method [25]. Actually, the proposed algorithm has been compared with the CDA as it has been of great inspiration for the development of cvxEDA, and is one of the widely used approach for EDA processing. Both methods employ the same impulse response function to model the sweating process, but the cvxEDA approach introduces 1) the ideas of modeling the phasic component as IIR, 2) solving a regularized quadratic-programming problem for EDA decomposition, and 3) using a constrained estimation to enforce nonnegativity of the SMNA driver signal. These three important features allowed us to obtain a sparse nonnegative representation and a greater computational efficiency for EDA processing.

One of the main issue in the EDA analysis concerns the absence of an universally accepted protocol for the validation of EDA analysis algorithms. Unless the sympathetic nerve activity is also recorded through microneurography, we can equally ascribe a failure detection of an SCR after the occurrence of a stimulus to a low sensitivity of the algorithm or to the inability of the stimulus to consistently elicit a phasic response.In this work, quantitative statistics of cvxEDA and CDA comparison were expressed in terms of time of execution of the algorithm, memory usage, and statistical significance in discerning affective stimuli along the force and velocity dimensions. We aimed to effectively discern caressing force and velocity levels, as elicited through a wearable haptic system [3]. EDA recordings were gathered from 32 healthy subjects ( 16 females), undergoing affective haptic elicitation through a wearable system able to convey caress-like stimuli by means of two motors, which stretch a strip of fabric. Six kinds of stimuli were randomly administered in time, being comprised of combinations of three velocities and two forces levels.

Concerning the study of the statistical power of the EDA features, for the caressing force dimension, only the diffTonic feature (computed from both cvxEDA and CDA outputs) was significantly different between the two caressing force levels. Moreover, higher caressing force was associated with higher feature values. This means that the higher the intensity of caressing, the higher is the tonic level of EDA after such a cutaneous stimulus. This is a reasonable behavior, being in line with typical physiological dynamics associated to EDA [15]. However, it is worthwhile noting that the cvxEDA provided tonic features with more discriminant power (i.e., lower $p$-values) than the CDA approach.

Along the caressing velocity dimension, experimental results demonstrated that cvxEDA modeling outperforms CDA approach. Features from the sparse phasic components of EDA, in fact, were able to discern all of the differences between caressing velocity levels. Phasic components estimated from CDA, instead, were not able to discern between $V 2$ and $V 3$, and were always associated with higher $p$-values than the cvxEDA ones (see Tables IV and V). Importantly, increasing monotonic trends among caressing velocities were associated to cvxEDArelated phasic features. Therefore, it is possible to conclude that cvxEDA modeling approach provides feature values able to automatically assess caressing stimuli in a force-velocity space.

We also demonstrated that the cvxEDA approach is particularly suitable for implementations in embedded computing systems. Computational performance analysis, in fact, demonstrated that the execution time of the cvxEDA algorithm linearly increases with the length of the acquisition, whereas processing time of CDA model tends to grow superlinearly (see Fig. 6). This is reasonably due to the CDA optimization stage [15]. In the cvxEDA approach, thanks to the use of an IIR representation of the IRF, the computational time is reduced respect to the AR modeling [29]. More specifically, for all practical purposes, the computational cost of the cvxEDA algorithm is dominated by the ARMA representation of the phasic activity within the constrained minimization QP convex problem. Both $M$ and $A$, in equation 7, are $N \times N$ tridiagonal matrices, i.e., sparse matrices with $O(N)$ nonzero elements. Whenever a sparse solver is employed, i.e., a solver that can exploit the sparsity of the problem, space complexity and time complexity are $O(N)$, i.e., they scale linearly with the length of the $\mathrm{SC}$ time series. Of 
note, a crucial running-time factor is the use of a good solver for the optimization problem. For example, in MATLAB language, the use of CVX toolbox [39] allows the choice among several solvers in addition to the MATLAB default (implemented in this version of the algorithm), that could further improve the performance.

Moreover, taking advantage of the sparse nature of its phasic components, cvxEDA-derived outputs needed significantly lower storage values than the CDA model, thus being more suitable for the implementation in wearable monitoring systems than CDA. Furthermore, it is worthwhile noting that the cvxEDA approach needs to solve a convex optimization problem, thus always guaranteeing to find the globally optimal solution. Moreover, it is worth noticing that the degree of sparsity of the cvxEDA-based phasic components depends on the number of peaks, i.e., number of stimulus responses, occurring in a given recording. A sparse representation is more convenient if there are a high number of zeros in the matrix or vector to be saved. Assuming a Poisson distribution of the nerve firing rate, we indirectly guarantee a good sparsity level. Therefore, differences in memory storage between the CDA and cvxEDA models could be minimized even in other experimental protocols involving high-frequency stimuli.

Findings of this study can be profitably exploited in the field of affective haptics or, more in general, wearable haptic devices [1]. These systems, in fact, require processing algorithms with low-computational cost and low-memory consumption, in order to effectively augment communication, interaction, and cooperation between human and robots. Regarding the affective part of caressing stimuli used in this study, in a previous endeavor, we demonstrated how caressing force and velocity levels relate to perceived arousal (the emotional intensity) and valence (the emotional pleasantness) levels of emotions [40]. Specifically, subjects' self-ratings revealed that caresses performed at low force and low velocity are perceived as more pleasant and less arousing than others [8], [9], [40].

Possible challenging application fields can be related to assistive devices and rehabilitation, e.g., with patients with severe brain damages, which can be in one of several states now collectively known as disorders of consciousness (DOC). Indeed, the treatment of these patients is often driven by experience, expertise, and intuition of the clinicians, but there is no standardized approach in order to investigate if some perceptual channels (such as touch, which was proven to communicate distinct emotions as discussed in the Introduction) are still active and can be used to communicate with them. DOC assessment and rehabilitation will benefit from wearable affective haptic systems, like the one described in this paper, together with the novel here presented processing approach, thus enabling remote control of the stimulation (e.g., coming from relatives) as well as reliable affective-inspired actuations. Finally, the cvxEDA algorithm could be also profitably used in conjunction with wearable sensing systems like the one in [13] to assess mental and physical stress [41], key parameters to monitor during the course of a rehabilitation intervention or for the use of a prosthesis.

Future works will focus on an actual implementation of the proposed cvxEDA algorithm in different portable/wearable devices, allowing the study of EDA signatures in a more naturalistic environment. Moreover, future work will include a gender analysis.

\section{REFERENCES}

[1] L. Meli, S. Scheggi, C. Pacchierotti, and D. Prattichizzo, "Wearable haptics and hand tracking via an RGB-D camera for immersive tactile experiences," in Proc. ACM SIGGRAPH Posters, 2014, Art. no. 56.

[2] D. Tsetserukou, A. Neviarouskaya, H. Prendinger, N. Kawakami, and S. Tachi, "Affective haptics in emotional communication," in Proc. 3rd Int. Conf. Affective Comput. Intell. Interaction Workshops, 2009, pp. 1-6.

[3] M. Bianchi et al., "Design and preliminary affective characterization of a novel fabric-based tactile display," in Proc. IEEE Haptics Symp., 2014, pp. 591-596.

[4] J. Chatel-Goldman, M. Congedo, C. Jutten, and J.-L. Schwartz, "Touch increases autonomic coupling between romantic partners," Frontiers Behav. Neurosci., vol. 8, 2014, Art. no. 95.

[5] J. Liljencrantz and H. Olausson, "Tactile C fibers and their contributions to pleasant sensations and to tactile allodynia," Frontiers Behav. Neurosci., vol. 8, 2014, Art. no. 37.

[6] Y. Zotterman, "Touch, pain and tickling: An electro-physiological investigation on cutaneous sensory nerves," J. Physiol., vol. 95, no. 1, pp. 1-28, 1939.

[7] E. T. Rolls, "The affective and cognitive processing of touch, oral texture, and temperature in the brain," Neurosci. Biobehav. Rev., vol. 34, no. 2, pp. $237-245,2010$.

[8] C. Triscoli, H. Olausson, U. Sailer, H. Ignell, and I. Croy, "Ct-optimized skin stroking delivered by hand or robot is comparable," Frontiers Behav., vol. 7, 2013, Art. no. 208.

[9] L. S. Löken, J. Wessberg, F. McGlone, and H. Olausson, "Coding of pleasant touch by unmyelinated afferents in humans," Nature Neurosci., vol. 12 , no. 5, pp. 547-548, 2009.

[10] L. S. Löken, M. Evert, and J. Wessberg, "Pleasantness of touch in human glabrous and hairy skin: Order effects on affective ratings," Brain Res., vol. 1417, pp. 9-15, 2011.

[11] A. Klöcker, C. M. Oddo, D. Camboni, M. Penta, and J.-L. Thonnard "Physical factors influencing pleasant touch during passive fingertip stimulation," PLOS One, vol. 9, no. 7, 2014, Art. no. e101361.

[12] R. A. Calvo and S. D'Mello, "Affect detection: An interdisciplinary review of models, methods, and their applications," IEEE Trans. Affective Comput., vol. 1, no. 1, pp. 18-37, Jan. 2010.

[13] A. Lanatà, G. Valenza, and E. P. Scilingo, "A novel eda glove based on textile-integrated electrodes for affective computing," Med. Biol. Eng. Comput., vol. 50, no. 11, pp. 1163-1172, 2012.

[14] A. Lanatà et al., "How the autonomic nervous system and driving style change with incremental stressing conditions during simulated driving,' IEEE Trans. Intell. Transp. Syst., vol. 16, no. 3, pp. 1505-1517, Jun. 2015.

[15] W. Boucsein, Electrodermal Activity. New York, NY, USA: Springer, 2012.

[16] H. Olausson et al., "Functional role of unmyelinated tactile afferents in human hairy skin: Sympathetic response and perceptual localization," Exp. Brain Res., vol. 184, no. 1, pp. 135-140, 2008.

[17] T. L. Chen, C.-H. A. King, A. L. Thomaz, and C. C. Kemp, "An investigation of responses to robot-initiated touch in a nursing context," Int. J. Social Robot., vol. 6, no. 1, pp. 141-161, 2014.

[18] H. Storm, "Changes in skin conductance as a tool to monitor nociceptive stimulation and pain," Curr. Opin. Anesthesiol., vol. 21, no. 6, pp. 796804, 2008.

[19] B. Hellerud and H. Storm, "Skin conductance and behaviour during sensory stimulation of preterm and term infants," Early Human Develop., vol. 70, no. 1, pp. 35-46, 2002.

[20] S. Ocklenburg, N. Rüther, J. Peterburs, M. Pinnow, and O. Güntürkün, "Laterality in the rubber hand illusion," Laterality, vol. 16, no. 2, pp. 174$187,2011$.

[21] M. D'Alonzo and C. Cipriani, "Vibrotactile sensory substitution elicits feeling of ownership of an alien hand," PLOS One, vol. 7, 2012, Art. no. e50756.

[22] I. Keller, A. Hulsdunk, and F. Muller, "The influence of acoustic and tactile stimulation on vegetative parameters and eeg in persistent vegetative state," Functional Neurol., vol. 22, no. 3, pp. 159-164, 2007.

[23] F. H. Wilhelm, A. S. Kochar, W. T. Roth, and J. J. Gross, "Social anxiety and response to touch: Incongruence between self-evaluative and physiological reactions," Biol. Psychol., vol. 58, no. 3, pp. 181-202, 2001. 
[24] A. Greco, G. Valenza, A. Lanata, E. P. Scilingo, and L. Citi, "cvxEDA: A convex optimization approach to electrodermal activity processing," IEEE Trans. Biomed. Eng., vol. 63, no. 4, pp. 797-804, Apr. 2016.

[25] T. Chaspari, A. Tsiartas, L. Stein, S. Cermak, and S. S. Narayanan, "Sparse representation of electrodermal activity with knowledge-driven dictionaries," IEEE Trans. Biomed. Eng., vol. 62, no. 3, pp. 960-971, Mar. 2015.

[26] M. Benedek and C. Kaernbach, "Decomposition of skin conductance data by means of nonnegative deconvolution," Psychophysiology, vol. 47, no. 4, pp. 647-658, 2010.

[27] A. Ishchenko and P. Shev'ev, "Automated complex for multiparameter analysis of the galvanic skin response signal," Biomed. Eng., vol. 23, no. 3, pp. 113-117, 1989.

[28] A. Greco, G. Valenza, A. Lanata, G. Rota, and E. Scilingo, "Electrodermal activity in bipolar patients during affective elicitation," IEEE J. Biomed. Health Informat., vol. 18, no. 6, pp. 1865-1873, Nov. 2014.

[29] A. Greco, A. Lanata, G. Valenza, E. P. Scilingo, and L. Citi, "Electrodermal activity processing: A convex optimization approach," in Proc. 36th Annu. IEEE Int. Conf. Eng. Med. Biol. Soc., 2014, pp. 2290-2293.

[30] A. Greco, A. Lanata, G. Valenza, G. Rota, N. Vanello, and E. P. Scilingo, "On the deconvolution analysis of electrodermal activity in bipolar patients," in Proc. IEEE Annu. Int. Conf. Eng. Med. Biol. Soc., 2012, pp. 6691-6694.

[31] W. T. Roth, M. E. Dawson, and D. L. Filion, "Publication recommendations for electrodermal measurements," Psychophysiology, vol. 49, pp. 1017-1034, 2012.

[32] M. Benedek and C. Kaernbach, "A continuous measure of phasic electrodermal activity," J. Neurosci. Methods, vol. 190, no. 1, pp. 80-91, 2010.

[33] E. R. Garrett, "The bateman function revisited: A critical reevaluation of the quantitative expressions to characterize concentrations in the one compartment body model as a function of time with first-order invasion and first-order elimination," J. Pharmacokinetics Biopharmaceutics, vol. 22, no. 2, pp. 103-128, 1994.

[34] D. Alexander, C. Trengove, P. Johnston, T. Cooper, J. August, and E. Gordon, "Separating individual skin conductance responses in a short interstimulus-interval paradigm," J. Neurosci. Methods, vol. 146, no. 1, pp. 116-123, 2005.

[35] C. Karenbach, "Ledalab-A software package for the analysis of phasic electrodermal activity," Allgemeine Psychologie, Institut für Psychologie, Freiburg, Germany, Tech. Rep., 2005.

[36] D. R. Bach, K. J. Friston, and R. J. Dolan, "An improved algorithm for model-based analysis of evoked skin conductance responses," Biol. Psychol., vol. 94, no. 3, pp. 490-497, 2013.

[37] D. R. Bach and M. Staib, "A matching pursuit algorithm for inferring tonic sympathetic arousal from spontaneous skin conductance fluctuations," Psychophysiology, vol. 52, pp. 1106-1112, 2015.

[38] C. L. Lim et al., "Decomposing skin conductance into tonic and phasic components," Int. J. Psychophysiol., vol. 25, no. 2, pp. 97-109, 1997.

[39] M. Grant, S. Boyd, and Y. Ye, "Cvx: Matlab software for disciplined convex programming," 2008.

[40] A. Greco, G. Valenza, M. Nardelli, A. Lanata, M. Bianchi, and E. P. Scilingo, "Electrodermal activity analysis during affective haptic elicitation," in Proc. IEEE Annu. Int. Conf. Eng. Med. Biol. Soc., 2015, pp. 5777 5780.

[41] R. Fernandez and R. W. Picard, "Modeling drivers' speech under stress," Speech Commun., vol. 40, no. 1, pp. 145-159, 2003.

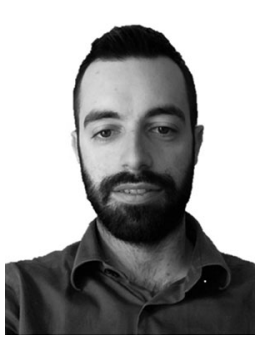

Alberto Greco (S'13-M'16) received the Graduate degree in biomedical engineering and the Ph.D. degree in automatics, robotics, and bioengineering from the University of Pisa, Pisa, Italy, in 2010 and 2015, respectively.

He is currently a Research Fellow with Research Center "E. Piaggio," University of Pisa, and he was a Visiting Fellow at the University of Essex, U.K., in 2014. His main research interests include physiological modeling, wearable monitoring systems, and biomedical signal processing. Applications include affective computing and the assessment of mood and consciousness disorders.

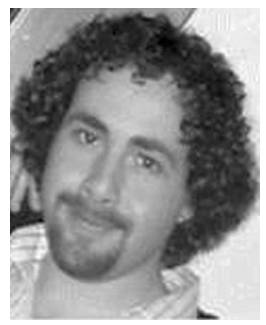

Gaetano Valenza (S'10-M'12) received the Ph.D degree in automation, robotics, and bioengineering from the University of Pisa, Pisa, Italy, in 2012.

He is currently a Research Fellow at the University of Pisa, and Harvard Medical School/Massachusetts General Hospital, Boston, MA, USA. His research interests include statistical and nonlinear biomedical signal and image processing, cardiovascular and neural modeling, and wearable systems for physiological monitoring. He is the author of more than 90 international scientific contributions in these fields, and is currently an Associate Editor of the Scientific Reports.

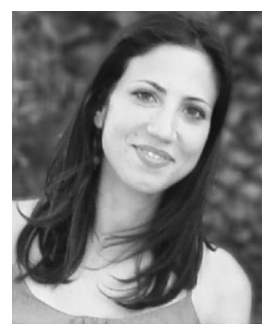

consciousness.

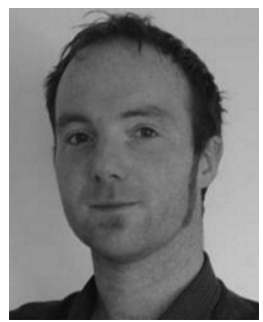

Matteo Bianchi (M'12) received the Ph.D. degree in automation, robotics, and bioengineering from the University of Pisa, Pisa, Italy, in 2012.

$\mathrm{He}$ is currently a Research Fellow at the Istituto Italiano di Tecnologia, Genoa, Italy, and the University of Pisa, and a Research Affiliate with the Mayo Clinic, Rochester, MN, USA. His research interests include haptic interface design, with applications in medical robotics and assistive/affective humanrobot interaction, human and robotic hands, optimal sensing, human-inspired control for soft robots, psychophysics, and mathematical modeling of the sense of touch and human manipulation. He is an author of contributions to international conferences and journals and has received several national and international awards in these fields.

Dr. Bianchi serves as a Reviewer and a Member of the Editorial Boards and organizing committees of international journals and conferences.

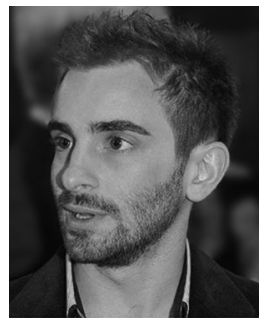

Luca Citi (S'06-M'09) received the Graduate degree in electronic engineering with a major in biomedical engineering from the University of Florence, Florence, Italy, in 2004, and the Ph.D. degree in biorobotics science and engineering from the Scuola Superiore Sant'Anna, Pisa, Italy, and Institutions, Markets, Technologies Lucca, Lucca, Italy, in 2009.

After working as a Postdoctoral Researcher at Massachusetts General Hospital/Harvard Medical School, Boston, MA, USA, he is currently a Lecturer (Assistant Professor) at the University of Essex, Colchester, U.K. His main research interests include signal processing and machine learning applied to physiological signals, computational neuroscience, and neural interfaces.

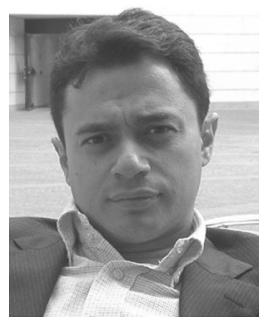

Enzo Pasquale Scilingo (M'10) received the Ph.D. degree in bioengineering from the Politecnico di Milano, Milan, Italy, in 1998.

$\mathrm{He}$ is an Associate Professor of electronic and information bioengineering at the University of Pisa, Pisa, Italy. He is engaged in several teaching activities and he is the Supervisor of several Ph.D. students. He coordinated the European project EC-FP7-ICT247777 "PSYCHE_Personalised monitoring SYstems for Care in mental Health." His main research interests include wearable monitoring systems, humancomputer interfaces, biomedical and biomechanical signal processing, modeling, control, and instrumentation. He is the author of more than 150 papers published peer-reviewed journals, contributions to international conferences, and chapters in international books. 